\title{
Seduction by Sensitivity: Reality, Illusion, or Delusion? The Challenge of Assessing Outcomes after PSMA Imaging Selection of Patients for Treatment
}

\author{
Rodney J. Hicks ${ }^{1,2}$, Declan G. Murphy ${ }^{2,3}$, and Scott G. Williams ${ }^{4}$ \\ ${ }^{I}$ Cancer Imaging, the Peter MacCallum Cancer Centre, Melbourne, Australia; ${ }^{2}$ The Sir Peter MacCallum Department of Oncology, \\ the University of Melbourne, Parkville, Australia; ${ }^{3}$ Division of Surgical Oncology, the Peter MacCallum Cancer Centre, Melbourne, \\ Australia; and ${ }^{4}$ Division of Radiation Oncology, the Peter MacCallum Cancer Centre, Melbourne, Australia
}

See the associated article on page 1972.

\section{$\mathbf{P}$}

rostate-specific membrane antigen (PSMA) has long been recognized to be an attractive target for evaluating prostate cancer (1). Over the past 2 decades, several groups have worked to develop imaging agents capable of leveraging the high expression of this target in most clinically significant prostate cancer and its relatively low expression in most other pathologic processes (2). These characteristics potentially provide the optimal conditions for both sensitive and specific prostate cancer imaging. Several groups have progressively refined synthesis of compounds that bind the extracellular domain of PSMA and are internalized through endocytosis. The most widely adopted and best validated of these agents has been ${ }^{68} \mathrm{Ga}$-PSMA-11 (3). This agent is not currently approved by the Food and Drug Administration but its use in other jurisdictions, including Europe and Australia, has been enabled by extemporaneous compounding regulations that apply within hospital environments. ${ }^{68} \mathrm{Ga}-\mathrm{PSMA}-11$ is now the subject of investigational new drug applications at multiple institutions in the United States, including a multicenter trial run by the Clinical Trials Network of the Society of Nuclear Medicine and Molecular Imaging. Other PSMA radiotracers are now becoming available. These include ${ }^{68} \mathrm{Ga}$-PSMA-I\&T (4) and ${ }^{18} \mathrm{~F}$ agents such as ${ }^{18}$ F-DCFPyL (5) and ${ }^{18}$ F-PSMA-1007 (6). Although data comparing these various agents that target PSMA are scarce, as a class, all seem to have a high sensitivity compared with other competing imaging modalities, particularly including the conventional staging techniques of abdominopelvic CT and bone scintigraphy (7). Direct comparison of PSMA and other molecular imaging techniques has also suggested superiority. For example, Morigi et al. demonstrated the ${ }^{68}$ Ga-PSMA-11 had a significantly higher detection rate than ${ }^{18} \mathrm{~F}$-fluoromethylcholine, especially at low prostate-specific antigen (PSA) levels (8).

Received Aug. 7, 2017; revision accepted Sep. 18, 2017.

For correspondence or reprints contact: Rodney J. Hicks, Cancer Imaging The Peter MacCallum Cancer Centre, Melbourne, Australia, 305 Grattan St., Melbourne, 3000 Victoria, Australia.

E-mail: rod.hicks@petermac.org

Published online Sep. 21, 2017.

COPYRIGHT (c) 2017 by the Society of Nuclear Medicine and Molecular Imaging. DOI: 10.2967/jnumed.117.198812
Despite our own long experience with ${ }^{18} \mathrm{~F}$-fluoromethylcholine PET/CT (9), with the very first ${ }^{68} \mathrm{Ga}$-PSMA-11 PET/CT performed at the Peter MacCallum Centre in July 2014, we knew that our diagnostic and treatment paradigms for prostate cancer would never be the same. The scan detected a node measuring less than $5 \mathrm{~mm}$ and an isolated rib metastasis that was not diagnostic on other imaging, thereby fundamentally changing the treatment plan for this patient. One of our group at the Peter MacCallum Centre commented that it was like seeing the tide rushing out and knowing that a tsunami was coming. This prediction has been realized over the succeeding $3 \mathrm{y}$. Such has been the incredible appetite for these scans, almost every PET/CT facility in Australia now offers PSMA imaging despite lack of government reimbursement. In our own facility, the growth in demand has outstripped our ability to supply scans in a timely manner given the constraints of ${ }^{68} \mathrm{Ga}$ generator-based radiopharmaceutical production. Consequently, we have migrated our program primarily to use of ${ }^{18} \mathrm{~F}$-DCFPyL. The ability to produce large activities of ${ }^{18} \mathrm{~F}$ agents allows more efficient and convenient distribution and use of this agent as well as facilitation of good manufacturing practice compliance. Whether the lower positron energy of ${ }^{18} \mathrm{~F}$ than ${ }^{68} \mathrm{Ga}$, which theoretically may enhance resolution, translates into higher sensitivity remains to be proven but preliminary evidence suggests diagnostic performance at least comparable to that of ${ }^{68} \mathrm{Ga}-\mathrm{PSMA}-11$ (10). Every day we are impressed by the performance of this technique in detecting otherwise occult sites of disease. The seduction of such sensitive imaging is pervasively felt in multidisciplinary meetings where an increasingly common retort to address diagnostic or management uncertainty is, "Let's get a PSMA scan."

PSMA imaging is probably the most accurate imaging technique that we have for detection of prostate cancer. The ability to detect involvement of small nodes is important because almost $80 \%$ of histopathologically confirmed nodal metastases have been found to occur in nodes smaller than the morphologic threshold of $8 \mathrm{~mm}$ (11). This has specific relevance to the planning of salvage radiotherapy (SRT) for biochemical failure. In a recent study involving 100 patients with low-level biochemical failure (median PSA, $1.0 \mathrm{ng} / \mathrm{mL}$ ), tumor recurrence was detected in $76 \%$ of cases by PSMA PET/CT (12). Of these sites, $80 \%$ had no morphologic correlate on CT or MRI and their detection led to a change in stage in $43 \%$ of cases. Similarly, another recent study involving 31 patients found suggestive foci in $87 \%$ with negative conventional imaging with $58 \%$ of the sites detected being in locations that would not have been covered in conventional radiotherapy fields (13). Although these data are impressive, we should not succumb 
to the illusion that this technique is perfect. Clearly, all sites of disease are not detected. In a series of patients with pathologic correlation, ${ }^{68} \mathrm{Ga}$-PSMA-11 was false-negative in just over $20 \%$ of cases (14). Although this was substantially superior to CT or MRI, which were false-negative in more than $70 \%$ of the same cases, these findings have implications for management decisions that might flow from a negative scan and for scans that indicate limited sites of metastatic disease, which might be amenable to aggressive locoregional therapies such as stereotactic radiotherapy. With increasing use of PSMA PET/CT, we have become aware that oligometastatic prostate cancer is largely a delusion based on the insensitivity of conventional imaging. The whole concept of oligometastatic disease has its proponents and detractors (15). We need to ensure that we do not subscribe to wishful thinking in interpreting negative PSMA scans or selecting to treat or not treat patients based on such a result.

In the current edition of the Journal of Nuclear Medicine, Emmett et al. (16) report on outcomes in patients receiving SRT for PSA relapse and particularly on the prognostic significance of a negative ${ }^{68} \mathrm{Ga}-$ PSMA-11 scan in this setting. In this series, involving 164 men who were considered eligible for SRT for biochemical failure after radical prostatectomy based on PSA readings between 0.05 and $1.0 \mathrm{ng} / \mathrm{mL}$, almost $40 \%$ of patients had a negative scan. Indeed, in those with a PSA of less than $0.2 \mathrm{ng} / \mathrm{mL}$, this rate increased to $50 \%$. After ${ }^{68} \mathrm{Ga}$-PSMA-11 PET/CT, 24 patients began androgen-deprivation therapy, presumably for distant disease, leaving 140 men who apparently remained candidates for SRT. For reasons that are not clear from the paper, SRT seems to have been influenced by factors other than just the PSMA result. Although all 36 men with disease confined to the prostate fossa on PSMA imaging were treated, most but not all those with involved nodes $(88 \%)$ and a substantial proportion of those with distant disease $(71 \%)$ were also treated. The latter group presumably had oligometastatic disease. Importantly, only 44\% $(n=27 / 60)$ of those with a negative PSMA PET went on to receive SRT. Although, this provided an opportunity to assess outcomes according to treatment delivered, the possibility that selection bias underlies the observed heterogeneity in treatment cannot be excluded. Nevertheless, in a novel finding alluded to in the title of the paper, they found an $85 \%$ PSA response rate in men with a negative PSMA who received SRT compared with further PSA increase in $65 \%$ of those not treated. At first glance, this suggests that a negative PSMA PET/CT identifies a group of men who particularly benefit from SRT and argues for use of SRT despite a negative PSMA.

Before accepting this premise, we need to consider reasons why PSMA might be negative in such patients and how these factors might influence prognosis independent of treatment or treatment response. First, it is evident that tumor burden, as reflected by PSA levels, seems to be an important predictor of PSMA scan positivity. In a large series of patients $(n=248)$ with biochemical recurrence after radical prostatectomy, although close to $90 \%$ had a positive PSMA scan, when the PSA was $0.2-<0.5 \mathrm{ng} / \mathrm{mL}$, this rate fell to $58 \%$ (17). Similarly, in a Turkish multicenter study involving 31 patients, the mean diameter of nodes that were false-negative on PSMA PET/CT was only $3.9 \mathrm{~mm}$ (18), which confirmed earlier results indicating that those pathologically involved nodes that were not detected were significantly smaller than nodes that were (19). Thus, it is likely that those patients with a negative scan have a smaller volume of disease than those with a positive scan. Consequently, their PSA levels may be more likely to fall back within the range specified for response by
Emmett et al. $(16)(<0.1 \mathrm{ng} / \mathrm{mL})$ even if some of their disease were to lie outside the treatment volume. Because of lag-time bias, a smaller volume of disease would likely take longer to manifest evidence of treatment failure. In this context, a median follow-up of 10 mo in their study is almost certainly too short to determine the overall survival benefit of SRT in such patients. The only robust means to determine outcome in prostate cancer treated with SRT is to prospectively assess the lack of failure over time, which in prostate cancer can mean a follow-up duration of years (or decades in the case of clinical failure endpoints). Short of this, use of a validated and widely accepted instrument (such as PSA $\geq$ 0.4 and rising) is the only reliable contemporary indicator of outcome (20). The response criteria proposed in the current report must be considered exploratory, especially because a fall in PSA alone cannot be relied on to determine longer-term outcomes (21). As with patients with measurable but low-level thyroglobulin levels after thyroidectomy, it is possible that a low but measurable PSA level may reflect residual prostatic tissue despite prostatectomy (22). The likelihood of significant residual normal tissue appears to be greater with conventional than with robotic radical prostatectomy (23). Treatment of such tissue by SRT may serve to reduce PSA levels while regrowth from this remnant over time in the absence of treatment may contribute to rising PSA levels, but in neither situation would this contribute to long-term outcome.

Second, tumor biology seems to be an important factor in determining PSMA expression. Studies suggest that tumor grade influences the likelihood of a positive scan with increasing Gleason score associated with higher PSMA expression (24). In the primary staging setting, the likelihood of a positive PSMA scan was related to both Gleason score and PSA levels (25). Further, preclinical studies indicate that folate may decrease the invasiveness of prostate cancer cells expressing PSMA (26), probably reflecting the function of PSMA as a folate hydrolase and suggesting that cases with high PSMA may be more likely to metastasize. Thus, a negative scan may indicate a lower grade of disease and a lower likelihood of disease existing beyond the prostate bed, the target of conventionally planned SRT. Although this might influence the likelihood of response, it might also indicate a more indolent disease process in which an observational strategy may limit side effects without adversely affecting survival. Consistent with this hypothesis, PSA doubling time has also been shown to be a predictive factor for a positive PSMA PET/CT $(27,28)$. An exception to the general rule that a negative scan implies either small volume or low-grade prostate cancer is the observation that neuroendocrine differentiation can be associated with low PSMA expression (29), and yet this is known to be an adverse prognostic group and can have low PSA levels (30). Because such disease may be missed on PSMA imaging, the likelihood of disease not being adequately included in an SRT field would be increased and lead to treatment failure.

Finally, masking of disease close to urinary activity may serve to render PSMA imaging false-negative. This is a situation in which multiparametric MRI, particularly if performed on a hybrid PET/MRI, might improve sensitivity. A recent study of 119 patients with PSA relapse revealed that multiparametric MRI improved detection of local recurrence close to the bladder base compared with ${ }^{68}$ Ga-PSMA-11 (31). Recently described alternative approaches are to perform delayed imaging with or without diuretic administration (32) or to perform early imaging before urinary activity reaches the bladder (33). Low urinary clearance of ${ }^{18}$ F-PSMA-1007 may prove to be an advantage in this regard (6). Because this cause of falsenegative PSMA relates to prostate bed recurrence, it would not be 
unexpected that such patients would have a PSA response to SRT, but again the longer-term benefit of this is likely to be determined by the likelihood of undetected disease beyond the treatment field and the biology of the disease.

As recently discussed in the Journal of Nuclear Medicine (34), the major challenge confronting oncologists managing prostate cancer is identifying which patients harbor significant and nonsignificant recurrence. With most current treatment having a rather low success in curing patients, avoiding toxicity and maintaining quality of life are important objectives in treatment selection. The paper by Emmett et al. (16) reminds us that however sensitive a test appears to be, it is illusory. As Richard Feynman famously observed, there's plenty of room at the bottom. Neither should we delude ourselves that sensitive response criteria will necessarily translate into superior patient outcomes. We await with interest further studies that assess the long-term outcomes of patients with negative PSMA PET/CT studies on which clinicians elect not to treat and the possibility of randomized trials of observation versus SRT in this setting. As Mark Twain posited, "Supposing is good, but finding out is better."

\section{DISCLOSURE}

Professor Rodney Hicks is supported by a National Health and Medical Research Council Practitioner Fellowship (APP110805) and program grant (APP1053792). No other potential conflict of interest relevant to this article was reported.

\section{REFERENCES}

1. Silver DA, Pellicer I, Fair WR, Heston WD, Cordon-Cardo C. Prostate-specific membrane antigen expression in normal and malignant human tissues. Clin Cancer Res. 1997;3:81-85.

2. Maurer T, Eiber M, Schwaiger M, Gschwend JE. Current use of PSMA-PET in prostate cancer management. Nat Rev Urol. 2016;13:226-235.

3. Afshar-Oromieh A, Avtzi E, Giesel FL, et al. The diagnostic value of PET/CT imaging with the ${ }^{68} \mathrm{Ga}$-labelled PSMA ligand HBED-CC in the diagnosis of recurrent prostate cancer. Eur J Nucl Med Mol Imaging. 2015;42:197-209.

4. Herrmann K, Bluemel C, Weineisen $\mathrm{M}$, et al. Biodistribution and radiation dosimetry for a novel probe targeting prostate specific membrane antigen for imaging and therapy. J Nucl Med. 2015;56:855-861.

5. Szabo Z, Mena E, Rowe SP, et al. Initial evaluation of $\left[{ }^{18} \mathrm{~F}\right] \mathrm{DCFPyL}$ for prostatespecific membrane antigen (PSMA)-targeted PET imaging of prostate cancer. Mol Imaging Biol. 2015;17:565-574.

6. Giesel FL, Cardinale J, Schafer M, et al. ${ }^{18}$ F-labelled PSMA-1007 shows similarity in structure, biodistribution and tumour uptake to the theragnostic compound PSMA-617. Eur J Nucl Med Mol Imaging. 2016;43:1929-1930.

7. Perera M, Papa N, Christidis D, et al. Sensitivity, specificity, and predictors of positive ${ }^{68} \mathrm{Ga}$-prostate-specific membrane antigen positron emission tomography in advanced prostate cancer: a systematic review and meta-analysis. Eur Urol. 2016;70:926-937.

8. Morigi JJ, Stricker PD, van Leeuwen PJ, et al. Prospective comparison of ${ }^{18} \mathrm{~F}$ fluoromethylcholine versus ${ }^{68} \mathrm{Ga}$-PSMA PET/CT in prostate cancer patients who have rising PSA after curative treatment and are being considered for targeted therapy. J Nucl Med. 2015;56:1185-1190.

9. Beauregard JM, Williams SG, Degrado TR, Roselt P, Hicks RJ. Pilot comparison of F-fluorocholine and F-fluorodeoxyglucose PET/CT with conventional imaging in prostate cancer. J Med Imaging Radiat Oncol. 2010;54:325-332.

10. Dietlein F, Kobe C, Neubauer S, et al. PSA-stratified performance of ${ }^{18} \mathrm{~F}$ - and ${ }^{68} \mathrm{Ga}$-PSMA PET in patients with biochemical recurrence of prostate cancer. J Nucl Med. 2017;58:947-952.

11. Hövels AM, Heesakkers RA, Adang EM, et al. The diagnostic accuracy of CT and MRI in the staging of pelvic lymph nodes in patients with prostate cancer: a meta-analysis. Clin Radiol. 2008;63:387-395.
12. Habl G, Sauter K, Schiller K, et al. 68 Ga-PSMA-PET for radiation treatment planning in prostate cancer recurrences after surgery: individualized medicine or new standard in salvage treatment. Prostate. 2017;77:920-927.

13. Schiller K, Sauter K, Dewes S, et al. Patterns of failure after radical prostatectomy in prostate cancer: implications for radiation therapy planning after ${ }^{68} \mathrm{Ga}-\mathrm{PSMA}-$ PET imaging. Eur J Nucl Med Mol Imaging. 2017;44:1656-1662.

14. Rauscher I, Maurer T, Beer AJ, et al. Value of ${ }^{68} \mathrm{Ga}$-PSMA HBED-CC PET for the assessment of lymph node metastases in prostate cancer patients with biochemical recurrence: comparison with histopathology after salvage lymphadenectomy. J Nucl Med. 2016;57:1713-1719.

15. Palma DA, Salama JK, Lo SS, et al. The oligometastatic state: separating truth from wishful thinking. Nat Rev Clin Oncol. 2014;11:549-557.

16. Emmett L, van Leeuwen P, Nandurkar R, et al. Treatment outcomes from ${ }^{68} \mathrm{Ga}$-PSMA PET/CT-informed salvage radiation treatment in men with rising PSA after radical prostatectomy: prognostic value of a negative PSMA PET. J Nucl Med. 2017;58:1972-1976.

17. Eiber M, Maurer T, Souvatzoglou M, et al. Evaluation of hybrid ${ }^{68} \mathrm{Ga}-\mathrm{PSMA}$ ligand PET/CT in 248 patients with biochemical recurrence after radical prostatectomy. J Nucl Med. 2015;56:668-674.

18. Öbek C, Doganca T, Demirci E, et al. The accuracy of ${ }^{68} \mathrm{Ga}$-PSMA PET/CT in primary lymph node staging in high-risk prostate cancer. Eur J Nucl Med Mol Imaging. June 18, 2017 [Epub ahead of print].

19. Budäus L, Leyh-Bannurah SR, Salomon G, et al. Initial experience of ${ }^{68} \mathrm{Ga}-$ PSMA PET/CT imaging in high-risk prostate cancer patients prior to radical prostatectomy. Eur Urol. 2016;69:393-396.

20. Stephenson AJ, Kattan MW, Eastham JA, et al. Defining biochemical recurrence of prostate cancer after radical prostatectomy: a proposal for a standardized definition. J Clin Oncol. 2006;24:3973-3978.

21. Choo R, Hruby G, Hong J, et al. (IN)-efficacy of salvage radiotherapy for rising PSA or clinically isolated local recurrence after radical prostatectomy. Int J Radiat Oncol Biol Phys. 2002;53:269-276.

22. Ravery V. The significance of recurrent PSA after radical prostatectomy: benign versus malignant sources. Semin Urol Oncol. 1999;17:127-129.

23. Loeb S, Epstein JI, Ross AE, Schultz L, Humphreys EB, Jarow JP. Benign prostate glands at the bladder neck margin in robotic vs open radical prostatectomy. BJU Int. 2010;105:1446-1449.

24. Chang SS. Overview of prostate-specific membrane antigen. Rev Urol. 2004;6(suppl 10):S13-S18.

25. Uprimny C, Kroiss AS, Decristoforo C, et al. ${ }^{68} \mathrm{Ga}$-PSMA-11 PET/CT in primary staging of prostate cancer: PSA and Gleason score predict the intensity of tracer accumulation in the primary tumour. Eur J Nucl Med Mol Imaging. 2017;44: 941-949.

26. Yao V, Parwani A, Maier C, Heston WD, Bacich DJ. Moderate expression of prostate-specific membrane antigen, a tissue differentiation antigen and folate hydrolase, facilitates prostate carcinogenesis. Cancer Res. 2008;68:9070-9077.

27. Ceci F, Uprimny C, Nilica B, et al. ${ }^{68} \mathrm{Ga}$-PSMA PET/CT for restaging recurrent prostate cancer: which factors are associated with PET/CT detection rate? Eur J Nucl Med Mol Imaging. 2015;42:1284-1294.

28. Verburg FA, Pfister D, Heidenreich A, et al. Extent of disease in recurrent prostate cancer determined by $\left[{ }^{68} \mathrm{Ga}\right]$ PSMA-HBED-CC PET/CT in relation to PSA levels, PSA doubling time and Gleason score. Eur J Nucl Med Mol Imaging. 2016;43:397-403.

29. Tosoian JJ, Gorin MA, Rowe SP, et al. Correlation of PSMA-targeted ${ }^{18} \mathrm{~F}$ DCFPyL PET/CT findings with immunohistochemical and genomic data in a patient with metastatic neuroendocrine prostate cancer. Clin Genitourin Cancer. 2017;15:e65-e68.

30. Beltran H, Tomlins S, Aparicio A, et al. Aggressive variants of castrationresistant prostate cancer. Clin Cancer Res. 2014;20:2846-2850.

31. Freitag MT, Radtke JP, Afshar-Oromieh A, et al. Local recurrence of prostate cancer after radical prostatectomy is at risk to be missed in ${ }^{68} \mathrm{Ga}$-PSMA-11-PET of PET/CT and PET/MRI: comparison with mpMRI integrated in simultaneous PET/MRI. Eur J Nucl Med Mol Imaging. 2017;44:776-787.

32. Afshar-Oromieh A, Sattler LP, Mier W, et al. The clinical impact of additional late PET/CT imaging with ${ }^{68} \mathrm{Ga}$-PSMA-11 (HBED-CC) in the diagnosis of prostate cancer. J Nucl Med. 2017;58:750-755.

33. Uprimny C, Kroiss AS, Decristoforo C, et al. Early dynamic imaging in ${ }^{68} \mathrm{Ga}-$ PSMA-11 PET/CT allows discrimination of urinary bladder activity and prostate cancer lesions. Eur J Nucl Med Mol Imaging. 2017;44:765-775.

34. Ballas LK, de Castro Abreu AL, Quinn DI. What medical, urologic, and radiation oncologists want from molecular imaging of prostate cancer. $\mathrm{J} \mathrm{Nucl}$ Med. 2016;57:6S-12S 